\title{
PSP2, a gene encoding RGG-motif protein, is a novel suppressor of clathrin heavy chain deficiency
}

Debadrita Roy, Mani Garg and Purusharth I Rajyaguru*

Department of Biochemistry, Indian Institute of Science, C V Raman road, Bangalore - 560012

Author for correspondence: *Purusharth I Rajyaguru, Department of Biochemistry, Indian Institute of Science,

C V Raman road, Bangalore - 560012; email: rajyaguru@iisc.ac.in

Running title: RGG-motif proteins suppress clathrin heavy chain deficiency.

Keywords: Clathrin heavy chain, translation repressor, SCD6, PSP2, SBP1, DED1, DHH1, GBP2 and RGG-motif. 


\begin{abstract}
Clathrin, made up of the heavy- and light-chains, constitutes one of the most abundant protein in vesicles involved in intracellular protein trafficking and endocytosis. YPR129W, which encodes RGGmotif containing translation repressor was identified as a part of multi-gene construct (SCD6) that suppressed clathrin deficiency. However, the contribution of YPR129W alone in suppressing clathrin deficiency has not been documented. In this study we identify YPR129W as a necessary and sufficient gene in a multigene construct SCD6 that suppresses clathrin deficiency. Importantly, we identify cytoplasmic RGG-motif protein encoding gene PSP2 as a novel suppressor of clathrin deficiency. Three other RGG-motif protein encoding genes SBP1, DED1 and GBP2 do not suppress clathrin deficiency. $D H H 1$, a DEAD-box RNA helicase with translation repression activity also fails to rescue clathrin deficiency. $\alpha$-factor secretion assay suggests that suppression of clathrin deficiency by SCD6 and PSP2 is not mediated by the rescue of the trans-Golgi network (TGN) protein sorting defect observed in the absence of $\mathrm{CHC1}$. Detailed domain analysis of the two suppressors reveals that the RGG-motif of both Scd6 and Psp2 is important for suppressing clathrin deficiency. Additionally, the Lsm domain deletion as well as the arginine to alanine mutation in the arginine methylation defective (AMD) mutant render Scd6 defective in suppressing clathrin deficiency. Overall based on our results using SCD6 and PSP2 proteins, we identify a novel role of RGG-motif in suppressing clathrin deficiency. Since both the suppressors are RNA-binding granule-resident proteins, this study opens an exciting avenue for exploring the connection between clathrin function and cytoplasmic RNA metabolism.
\end{abstract}




\section{Introduction}

Clathrin is a protein of vital importance in cells. Clathrin coated vesicles (CCVs) play a key role in endocytosis and various intracellular trafficking. The triskelion, which is made up of the clathrin light chain and heavy chain monomers, forms a typical polygonal surface lattice on CCVs. Clathrin mediated endocytosis, which is highly conserved from yeast to humans, is the major pathway for the internalization of external and membrane molecules into the cell. There are clathrin-independent pathways, however without clathrin-mediated endocytosis, uptake is greatly reduced ${ }^{1-4}$. CCVs are also involved in the intracellular trafficking pathway. Deficiency of clathrin in unicellular organisms like yeast, amoebae and protozoa are associated with defects in intracellular trafficking and receptor-mediated endocytosis ${ }^{5,6}$.

In certain yeast strains, the deletion of the clathrin heavy chain-1 gene $(\mathrm{CHC} 1)$ renders cells inviable, whereas in others, cells are viable. This is owing to the presence of an independently segregating gene - suppressor of clathrin deficiency 1 (SCD1). Presence of the scd1-i allele leads to lethality in chc1 deficient cells, whereas the scd1-v allele allows growth, albeit poorly. Along with slow growth, chc1 $\Delta$ scd1-v cells have abnormal morphology, genetic instability, reduced endocytosis and mislocalization of endoproteases from the trans-Golgi network to the cell membrane ${ }^{7-12}$. SCD1 was recently identified to encode $P A L 2$, a protein involved in localizing to cortical patches with other endocytic factors, thereby facilitating endocytosis. It was found that scd1-i allele has a premature stop codon which results in a truncated version of wild type PAL2, encoded by scd1-v allele ${ }^{13}$. YEpSCD6 was one of the six multi-gene multicopy suppressors identified in a genetic screen ${ }^{14}$. This suppressor contains a total of 8 genes along with YPR129W. A personal communication on Saccharomyces genome database indicated YPR129W as a multicopy suppressor of clathrin deficiency (Gelperin et al. 1995, personal communication to SGD). YPR129W encodes an RGG-motif translation repressor protein that targets translation initiation factor elF4G1 ${ }^{15}$. We wanted to confirm its role in affecting clathrin function and further address the possible role of other RGGmotif containing RNA-binding proteins in affecting clathrin function. We hypothesized that other RGG-motif proteins could also affect clathrin function.

RGG-motif proteins contain single or multiple repeats (Table 1 ) of RGG/RGX (' $X$ ' being any residue). This motif is known to be involved in protein-nucleic acids and protein-protein interactions ${ }^{16}$. The RGG-motif containing proteins function in processes like transcription, cell cycle, apoptosis and synaptic plasticity. The RGG-motif constitutes the second most common class of domains/motifs that contribute to RNA-binding ${ }^{17}$. Recently, a subset of yeast RGG-motif proteins (such as Scd6, Sbp1, Npl3 and Ded1) have been implicated in translation control ${ }^{15,18}$ as these proteins bind conserved translation initiation factor elF4G through their RGG-motif to repress translation. Scd6 and Sbp1 are translation repressors and decapping activator proteins. Consistent with their role, both Scd6 and Sbp1 localize to RNA granules that are sites of translation repression and mRNA decay 15,19,20. PSP2 encodes a cytosolic RGG-motif (Table 1) containing protein originally identified as a suppressor of intron-splicing defects of a mutation in MRS2 and subsequently as a suppressor of conditional mutation in DNA POL $I$ in yeast ${ }^{21,22}$. Interestingly, Psp2 localizes to processing bodies during glucose deprivation ${ }^{19}$. DED1 encodes a DEAD-box RNA helicase and a RNA granule resident protein that can both promote and inhibit translation initiation in yeast ${ }^{23,24}$. GBP2 encodes a nucleocytoplasmic shuttling protein involved in telomere length modification ${ }^{25}$, mRNA export ${ }^{26-28}$ and mRNA quality control ${ }^{29}$. Despite being a nuclear protein, Gbp2 associates with polysomes and is a component of stress granules ${ }^{30,31}$ the significance of which remains to be understood. $\mathrm{DHH} 1$ 
encodes a DEAD-box RNA helicase that acts as a translation repressor and decapping activator ${ }^{32}$. It localizes to RNA granules and regulates P-body assembly in ATP-dependent manner ${ }^{33}$. In this work, we report that YPR129W is a bonafide suppressor of clathrin deficiency. We identify PSP2, a gene encoding RNA-binding and RGG-motif protein as a novel suppressor of clathrin deficiency. The RGGmotif of both Scd6 and Psp2 is important for suppression of clathrin deficiency. These results establish an unprecedented link between clathrin heavy chain function and granule-resident RGGmotif proteins. Our observations pave the way for exploring the mechanistic basis underlying this link in the future.

Table 1: Number of RGG/RGX in different proteins tested in this study

\begin{tabular}{|l|l|l|}
\hline $\begin{array}{l}\text { Protein (\# of amino } \\
\text { acids) }\end{array}$ & \# of RGG-/RGX- & Location of RGG-/RGX- motifs \\
\hline Scd6 (349) & $1 / 7$ & C-terminal \\
\hline Psp2 (593) & $4 / 10$ & C-terminal \\
\hline Sbp1 (294) & $8 / 5$ & Central \\
\hline Ded1 (604) & $4 / 4$ & Dispersed \\
\hline Gbp2 (427) & $4 / 6$ & Predominantly towards N-terminal \\
\hline
\end{tabular}

\section{RESULTS}

\section{YPR129W is necessary and sufficient to suppress clathrin deficiency}

YEpSCD6, a high copy number multi-gene plasmid was reported to be capable of rescuing inviability of clathrin heavy chain-deficient yeast strain expressing endogenous CHC1 under a galactoseinducible promoter ${ }^{14}$. This multi-gene construct contained 7 other genes along with YPR129W. Based on this study and the personal communication (Gelperin et al. 1995, personal communication to SGD), we were intrigued by the possible role of an RNA-binding translation repressor protein in suppressing clathrin heavy chain requirement and therefore wanted to indeed confirm the role of YPR129W in clathrin function. To test in our hands, the necessity and sufficiency of YPR129W, we created YEp $\triangle S C D 6$ (lacking YPR129W) and YEponlySCD6 (YEpoSCD6; expressing only YPR129W) by site directed mutagenesis. These plasmids (Table 3) were transformed into GAL1:CHC1 strain and the transformants were assayed for growth on glucose and galactose. We observed that YEp $\triangle S C D 6$ transformant grew as poorly as the empty vector control on glucose plate (Figure $1 \mathrm{~A}$ ) indicating that in the absence of SCD6 none of the other 7 genes could suppress the clathrin deficiency growth defect. Thus, SCD6 was necessary in the multi-gene construct to suppress clathrin deficiency mediated growth defect. YEpoSCD6 transformed cells grew in manner comparable to YEpSCD6 transformed cells suggesting that $S C D 6$ was sufficient to suppress the clathrin deficiency mediated growth defect (Figure 1A). In order to quantify the extent of rescue, a plating assay was carried out on YEP media, wherein after depleting Chc1, cells were plated on galactose and glucose followed by counting the colonies. Consistent with the growth assay, cells expressing YEpSCD6 and YEpoSCD 6 but not YEp $\triangle s c d 6$ could suppress clathrin deficiency. (Figure 1B). To further confirm its role, we cloned YPR129W in a $2 \mathrm{u}$ plasmid pGP564 with a C-terminal His-tag and checked its ability to suppress clathrin deficiency. We observed that this plasmid encoded SCD6 suppressed the clathrin deficiencymediated growth defect in a manner comparable to YEpSCD6 (Figure 1C). Based on these results we confirmed and established that YPR129W is a suppressor of clathrin deficiency. 


\section{PSP2 but no other RGG-motif protein encoding genes can suppress of clathrin deficiency defect}

SCD6 encodes RGG-motif containing protein which acts as a translation repressor. We wanted to check whether other genes encoding RGG-motif proteins and/or translation repressors can suppress clathrin deficiency. We therefore tested DED1, SBP1, DHH1, GBP2 and PSP2. Sbp1, Ded1 and Dhh1 can act as translation repressor proteins. Sbp1 and Ded1 (but not Dhh1) contains RGG-/RG- motifs which vary in number of repeats and location in the protein sequence (Table 1). Gbp2 is a shuttling RGG-motif protein which predominantly localizes to the nucleus. Psp2 is an RGG-motif containing protein, which localizes to RNA granules and has recently been reported to modulate translation of specific mRNAs involved in autophagy (Yin et al., 2019). Interestingly DED1, SBP1, DHH1 and GBP2 did not suppress the clathrin deficiency mediated growth defect (Figure 2A). Plating assay further confirmed the growth assay results (Figure 2B). Strikingly, PSP2 suppressed clathrin deficiency mediated growth defect (Figure $3 \mathrm{~A}$ ). Plating analysis followed by colony count confirmed the results obtained using growth assay (Figure 3B). The percentage rescue by PSP2 improved when the plating assay was performed on selective synthetic media plates likely due to increased retention of the plasmid (Supp. Figure 1). Based on these results we conclude that PSP2 is a novel suppressor of clathrin deficiency. These results also indicate that the genes encoding RGG-motif containing or translation repressor proteins in general do not suppress clathrin deficiency highlighting the specificity of suppression by SCD6 and PSP2.

\section{SCD6 and PSP2 do not rescue the trans-Golgi network (TGN) sorting function of the CHC1}

We next tested the ability of SCD6 and PSP2 to rescue the TGN sorting defects of $C H C 1$ depleted cells. MAT $\alpha$ cells are defective in the secretion of mature form of the mating pheromone $\alpha$-factor, in the absence of clathrin. This is due to the mis-localization of $\alpha$-factor processing enzymes from the trans-Golgi network to the plasma membrane ${ }^{9}$. As a result, MAT $\alpha$ cells with defective clathrin function do not inhibit the growth of MATa cells otherwise evident by a zone of growth inhibition (halo) in the halo assay. Since SCD6 and PSP2 rescue the growth defect phenotypes upon depletion of Chc1, we decided to test their contribution to $\alpha$-factor secretion. GAL1:CHC1 MAT $\alpha$ cells overexpressing plasmid encoding $C H C 1, S C D 6, S B P 1, P S P 2, G B P 2$ or DED1 were depleted of Chc1 by growing on glucose and spotted on a lawn of MATa cells expressing the sst1-2 allele (BJ3556 strain in Table 2). GAL1:CHC1 MATa cells overexpressing CHC1 from a plasmid form a clear zone of growth inhibition (halo) however such a zone of inhibition is absent for cells overexpressing any of the tested genes including SCD6 and PSP2 (Figure 4). We interpret this result to suggest that the suppression of clathrin deficiency growth defect by SCD6 and PSP2 does not involve complementation of the TGN function.

\section{RGG-motif plays a vital role in suppressing clathrin-deficiency}

Both SCD6 and PSP2 contain RGG-motif rich C-terminal region. SCD6 also contains an Lsm domain at its $\mathrm{N}$-terminus and a central FDF domain. PSP2 does not contain any other canonical domain/motif other than the RGG-motif. We investigated which domain of these proteins was responsible for the observed suppression of growth defect. To this end, we generated domain deletion constructs and assayed them for suppression of clathrin deficiency mediated growth defect. An RGG-deletion mutant of PSP2 was created and tested for its ability to suppress clathrin deficiency. We observed that the RGG-motif deletion mutant of PSP2 was defective in suppressing clathrin deficiency (Figure $5 A)$. Plating assays followed by colony counting further confirmed that the RGG-motif deletion mutant of PSP2 was defective in rescuing growth on the glucose media (Figure 5B and Supp. Figure 
1). We conclude that the RGG-motif of PSP2 plays an important role in suppressing clathrin deficiency.

The RGG-motif deletion construct of SCD6 was also highly defective in suppressing clathrin deficiency (Figure 6A). The Lsm domain deletion construct was also defective in suppressing clathrin deficiency albeit to slightly lesser extent than the RGG-motif deletion construct. Deletion of the FDF motif weakly affected the ability of the mutant to suppress clathrin deficiency. The RGG-motif (282348 residues) of SCD6 as annotated in literature ${ }^{34}$ comprises of RGG-rich sequences (282-310 residues) and QN-rich sequences (311-348 residues). Deletion of just the RGG-rich sequences (282310) (scd6RGG') resulted in a defect that was comparable to the deletion of 282-348 residues indicating that the RGG-rich sequence was indeed important for the rescue of clathrin deficiency. Based on these results we conclude that all the three domains affect the ability of SCD6 to suppress clathrin deficiency. RGG-motif is the least dispensable motif and FDF-domain is the most dispensable domain for suppressing clathrin deficiency. Plating experiments followed by colony counting confirmed the growth assay results (Figure 6B). Interestingly the FDF domain deletion mutant was also defective in suppressing clathrin deficiency in the plating assay which was not evident as much in the growth assay (Figure 5A). The differential behavior of this mutant in two different assays is interesting however the basis for this is unclear. Some of the arginine residues in the RGG-motif of Scd6 are methylated and this modification promotes the repression activity ${ }^{35}$. We tested the role of arginine residues of the RGG-motif in suppressing clathrin deficiency. We observed that the arginine methylation defective mutant (AMD; with $9 \mathrm{R}$ to $\mathrm{A}$ substitutions) failed to suppress the clathrin deficiency both in growth and plating assay (Figure $6 C \& D$ ). It is possible that arginine methylation of SCD6 RGG-motif could play a role in suppression of clathrin deficiency. Endogenous Scd6 is expressed in low copy number [1280 molecules/cell $\left.{ }^{36}\right]$ and we believe that its expression is highly regulated. Detection of Scd6 and its mutants expressed from pGP564 (Figure 6) using an anti-His antibody has been technically challenging. We however know that expression of both RGG-deletion and AMD mutant of Scd6 is not compromised when expressed under galactose-inducible promoter from a 2u plasmid (BG1805 vector) and detected using PAP reagent that detects the C-terminal ZZtag ${ }^{35}$. We also know that expression of Lsm domain deletion mutant is not compromised when expressed in the BG1805 vector (Parbin et al., under review). Overall these results indicate that the Scd6 RGG-motif and arginines in RGG-motif are important for suppression of clathrin deficiency. Interestingly the Lsm and the FDF (to a certain extent) domains also contribute to suppression of clathrin deficiency by Scd6.

\section{Discussion}

We provide evidence suggesting that two genes encoding RGG-motif proteins function as suppressors of clathrin deficiency. To the best of our knowledge this is the first peer-reviewed report implicating RGG-motif proteins in clathrin function. Our conclusion is based on the following observations, a) SCD6 acts as a suppressor of clathrin deficiency (Figure 1) b) PSP2 is a novel suppressor of clathrin deficiency (Figure 3) c) RGG-motifs of Psp2 and Scd6 are required for suppressing clathrin deficiency (Figure 5 and 6) d) Arginine residues of the Scd6 RGG-motif play an important role in suppressing clathrin deficiency (Figure 6C and D).

SCD6 was identified as one of the genes in a multi-gene construct that suppressed clathrin heavy chain deficiency, however the specific contribution of SCD6 remained to be confirmed. Our results prove that in the reported multi-gene construct, SCD6 is both necessary and sufficient to suppress clathrin deficiency mediated growth defect. This along with the observation that SCD6 cloned in a 
different vector also suppresses clathrin deficiency indicates that it is a genuine suppressor of clathrin deficiency. Reported roles of $S C D 6$ orthologs point towards a role of this family of proteins in endocytosis and/or trafficking. CAR-1 (worm ortholog) plays a role in maintaining ER organization as upon CAR-1 knockdown, ER is disorganized into large patches and thick strands ${ }^{37}$. Trailer hitch (Drosophila ortholog) localizes to ER-exit sites, which persist as large foci upon Tral knockdown ${ }^{38}$. Whether SCD6 functions along similar lines as its worm and fly ortholog is unclear.

Suppression of clathrin deficiency by PSP2 is a striking result. Psp2 is known to localize to RNA granules ${ }^{19}$ and upon overexpression, can rescue P-body formation in edc $3 \Delta / s m 4 \Delta c$ strain ${ }^{39}$. Scd6 binds TIF4631 (elF4G1) to repress translation and interestingly Psp2 is reported to physically interact with its paralog TIF4632 ${ }^{40}$. A recent report has indeed implicated Psp2-TIF4632 interaction in modulating translation of autophagy genes (Yin et al., 2019). Whether Psp2 can act as a translation repressor of specific mRNAs has not been explored. Requirement of the Psp2 RGG-motif in suppressing clathrin deficiency (Figure 5B) points toward a mechanism likely to be similar to that of Scd6 which needs to be tested further.

Lack of suppression by other RGG-motif proteins and translation repressors (Sbp1, Ded1, Dhh1 and Gbp2) highlights the specificity of Scd6 and Psp2 in clathrin function. It is unlikely that the contribution of Scd6 and Psp2 in clathrin function is dependent on the number of RGG-/RGX- repeats as Sbp1 contains more repeats than Scd6 (Table 1) but fails to suppress. Interestingly both Scd6 and Psp2 harbor RGG-motifs at its C-terminal but none of the other tested proteins including Sbp1 have RGG-motifs at their C-terminus.

Clathrin deficiency suppression mechanisms might likely fall into one of the following two categories: a) Suppressors that directly contribute to clathrin function in vesicular transport /endocytosis and b) Suppressors that indirectly increase tolerance to clathrin deficiency. SCD4 ${ }^{41}$ and $S C D 5^{11}$ belong to the former category that contribute directly to vesicular transport. SCD2 on the other hand belongs to latter category of suppressors. It was identified as UB/4 ${ }^{14}$ which likely suppresses clathrin deficiency defect by accelerating clearance of mis localized proteins We hypothesize that SCD6 and PSP2 might act in an indirect manner by altering translation to regulate the load of newly synthesized proteins on the trafficking machinery. This hypothesis is based on the following observations: 1) RGG-motif, which is required for translation repression activity of SCD6 $15,35,42$ is also required for suppressing clathrin deficiency defect by SCD6 and PSP2 2) SCD6 AMD mutant is defective in repressing translation as well as in suppressing clathrin deficiency 3) Lsm domain is required for the translation repression activity of Scd6 (Parbin et al., communicated) and also for suppressing clathrin deficiency. Several key experiments will be required in future to test this hypothesis. It is also possible that translation control of specific mRNAs involved in endocytosis and/or trafficking by Scd6 and perhaps Psp2 contributes to the suppression of clathrin deficiency. Identification of specific mRNA targets of Scd6 and Psp2 will provide important insights in this regard. Since Scd6 localizes to RNA granules in RGG-motif dependent manner and RNA granules are sites of translation repression and mRNA decay, we tested the localization of Scd6 upon clathrin depletion. We did not observe any significant difference in localization of Scd6 to foci (data not shown) suggesting that suppression is likely not mediated by changes in localization to foci.

LSM14 is the human ortholog of Scd6 ${ }^{34,43}$. The role of Scd6 identified in mRNA fate determination in yeast is conserved in humans. It is possible that LSM14 could contribute to clathrin mediated endocytosis and trafficking. This exciting possibility will be tested in future. Overall our results establish a new link between RGG-motif containing RNA-binding proteins and clathrin function. 
Analyzing the mechanistic basis of this link could lead to unraveling of the role of mRNA translation control pathway in clathrin mediated endocytosis. It will also be pertinent to test if the players contributing to endocytic/trafficking pathway including Chc1 are involved in modulating cytoplasmic mRNA fate. Our current work thus highlights several interesting research questions and addressing these questions will be an exciting future endeavor.

\section{Acknowledgements}

This work was predominantly supported by Department of Science and Technology grant (EMR/2017/001332) from the government of India. It was also supported by India Alliance DBTWellcome trust $(\mathrm{IA} / \mathrm{I} / 12 / 2 / 500625)$ and DBT-IISC partnership program (BT/PR27952INF/22/212/2018). DR was supported by DST-INSPIRE fellowship; MG is supported by University Grants Commission (UGC) fellowship. We are indebted to Sandra Lemmon (University of Miami) for helping us with various strains and reagents. We thank her for providing inputs at different stages of this work. We also sincerely thank Roy Parker (University of Colorado, Boulder) and Kenji Irie (University of Tsukuba) for sharing plasmids and strains.

\section{Competing Interests}

The authors declare no competing interest.

\section{Contributions}

PIR and DR designed the experiments; DR and MG performed the experiments; PIR, DR, and MG prepared the figures; PIR wrote the manuscript; PIR and MG revised the manuscript.

\section{Materials and methods}

Table 2: List of strains used in this study

\begin{tabular}{|l|l|l|l|}
\hline Name & Genotype & Description & Source \\
\hline GAL:CHC1 & $\begin{array}{l}\text { MATa GAL1:CHC1 scdl-i leu2 } \\
\text { ura3-52 trpl hisl GAL2 }\end{array}$ & $\begin{array}{l}\text { Wild type Saccharomyces } \\
\text { cerevisiae with inviable } \\
\text { allele of SCD1 and CHC1 } \\
\text { under galactose inducible } \\
\text { promoter }\end{array}$ & 14 \\
\hline BJ3556 & $\begin{array}{l}\text { MATa sst1-2 ade2-1 his6 met1 } \\
\text { cyh2 rme1 ura1 can1 }\end{array}$ & $\begin{array}{l}\text { Saccharomyces cerevisiae } \\
\text { with defective adaptation } \\
\text { to alpha factor response } \\
\text { pathway }\end{array}$ & 44 \\
\hline MATa & $\begin{array}{l}\text { MATa leu2 ura3-52 trp1 his3- } \\
\text { 200 }\end{array}$ & $\begin{array}{l}\text { Wild type Saccharomyces } \\
\text { cerevisiae with 'a' mating } \\
\text { type }\end{array}$ & 44 \\
\hline MATa & MATa leu2 ura3-52 trp1 his3- & $\begin{array}{l}\text { Wild type Saccharomyces } \\
\text { cerevisiae with 'alpha' } \\
\text { mating type }\end{array}$ & 44 \\
\hline
\end{tabular}

Table 3: List of plasmids used in this study

\begin{tabular}{|l|l|l|l|}
\hline Name & Description & Vector & Source \\
\hline YEp24 (empty vector) & $\begin{array}{l}\text { YEp24 library empty vector, } \\
\text { multicopy } 2 \mu \text { plasmid with URA3 }\end{array}$ & YEp24 & 14 \\
\hline
\end{tabular}




\begin{tabular}{|c|c|c|c|}
\hline & and ampicillin resistance genes & & \\
\hline YEpSCD6 & $\begin{array}{l}\text { YEp24 cloned with a fragment } \\
\text { from digested yeast genome } \\
\text { containing YPR129W along with } \\
\text { other flanking genes }\end{array}$ & YEp24 & 14 \\
\hline YEpoSCD6 & $\begin{array}{l}\text { Plasmid with only } S C D 6 \text { gene from } \\
\text { YEpSCD6, other flanking genes } \\
\text { deleted }\end{array}$ & YEp24 & This study \\
\hline YEp $\Delta s c d 6$ & $\begin{array}{l}\text { Plasmids retaining all genes } \\
\text { except } S C D 6 \text { from YEpSCD6 }\end{array}$ & YEp24 & This study \\
\hline pGP564 (empty vector) & $\begin{array}{l}\text { Yeast shuttle vector, multicopy } 2 \mu \\
\text { plasmid with } L E U 2 \text { and kanamycin } \\
\text { resistance genes }\end{array}$ & pGP564 & YGTC library, Lab Stock \\
\hline pGP SCD6 & $\begin{array}{l}\text { pGP564 containing SCD6 under its } \\
\text { own promoter. Along with the } \\
\text { ORF } 550 \text { nucleotides upstream } \\
\text { and } 225 \text { nucleotides downstream } \\
\text { are present. A 6X-His tag is } \\
\text { present before the stop codon of } \\
\text { SCD6. }\end{array}$ & pGP564 & This study \\
\hline pGP scd6 6 Lsm & $\begin{array}{l}\text { pGP SCD6 with Lsm domain } \\
\text { deletion: amino acids deleted: 2- } \\
93\end{array}$ & pGP564 & This study \\
\hline pGP scd6 $6 F D F$ & $\begin{array}{l}\text { pGP SCD6 with FDF domain } \\
\text { deletion; amino acids deleted: } \\
\text { 199-281 }\end{array}$ & pGP564 & This study \\
\hline pGP scd6 $6 R G G$ & $\begin{array}{l}\text { pGP SCD6 with RGG domain } \\
\text { (including QN rich part) deletion; } \\
\text { amino acids deleted: } 282-348\end{array}$ & pGP564 & This study \\
\hline pGP scd6 $6 R G G^{\prime}$ & $\begin{array}{l}\text { pGP SCD6 with RGG domain } \\
\text { (excluding QN rich part) deletion, } \\
\text { amino acids deletion: } 282-310\end{array}$ & pGP564 & This study \\
\hline pGP scd6AMD & $\begin{array}{l}\text { pGP SCD6 with R288A, R292A, } \\
\text { R294A, R298A, R301A, R304A, } \\
\text { R306A, R310A, R316A }\end{array}$ & pGP564 & This study \\
\hline pRS426 (empty vector) & $\begin{array}{l}\text { Yeast shuttle vector, multicopy } 2 \mu \\
\text { plasmid with URA3 and ampicillin } \\
\text { resistance genes }\end{array}$ & pRS426 & 20 \\
\hline pRS426 PSP2 & $\begin{array}{l}\text { pRS426 expressing PSP2 under its } \\
\text { own promoter }\end{array}$ & pRS426 & 39 \\
\hline $\mathrm{pRS} 426$ psp2 $2 \Delta G G$ & $\begin{array}{l}\text { pRS426 PSP2 with RGG domain } \\
\text { deletion; amino acids deleted: } \\
418-577\end{array}$ & pRS426 & This study \\
\hline $\mathrm{pRS426} D E D 1$ & $\begin{array}{l}\text { pRS } 426 \text { expressing } D E D 1 \text { under its } \\
\text { own promoter }\end{array}$ & pRS426 & 18 \\
\hline pRS426 SBP1 & $\begin{array}{l}\text { pRS426 expressing SBP1 under its } \\
\text { own promoter }\end{array}$ & pRS426 & 20 \\
\hline $\begin{array}{l}\text { YEplac195 (empty } \\
\text { vector) }\end{array}$ & $\begin{array}{l}\text { Yeast shuttle vector, multicopy } 2 \mu \\
\text { plasmid with URA3 and ampicillin } \\
\text { resistance genes }\end{array}$ & YEplac195 & 45 \\
\hline
\end{tabular}




\begin{tabular}{|l|l|l|l|}
\hline YEplac195 DHH1 & $\begin{array}{l}\text { YEplac195 expressing DHH1 under } \\
\text { its own promoter }\end{array}$ & YEplac195 & 45 \\
\hline Gbp2-GFP & $\begin{array}{l}\text { pPS1372 expressing GBP2-GFP } \\
\text { under its own promoter, } \\
\text { multicopy 2 } \mu \text { plasmid with LEU } \\
\text { and ampicillin resistance genes }\end{array}$ & pPS1372 & 29 \\
\hline
\end{tabular}

\section{Strains and Plasmids}

Strains and plasmids used in this study are enlisted in Table 2 and Table 3 respectively.

\section{Growth Assay}

Growth assays were performed to assess the ability of plasmids expressing desired genes to rescue clathrin deficiency mediated growth defect of the GAL:CHC1 strain on glucose media. In GAL:CHC1 strain, $\mathrm{CHC1}$ is under a galactose inducible promoter ${ }^{14}$ which results in a growth defect on glucose media. Transformants grown on galactose containing media $\left(10 \mathrm{OD}_{600}\right)$ were, serially diluted and spotted on the respective minimal media containing glucose and galactose plates. All the plated were incubated at $30^{\circ} \mathrm{C}$ and imaged after 2 days (galactose media) or 4-5 days (glucose media). Growth assays were performed at least three times with comparable results.

\section{Plating Assay}

After growing cells in YEP-glucose for more than 16 -20h, different GAL:CHC1 transformants were diluted to $0.001 \mathrm{OD}_{600}$. Three different volumes (50ul, 100ul and 200ul) were plated on YEPgalactose and glucose plates. The galactose containing plates were incubated for 4 days and glucose plates for 7 days before imaging. Colonies were counted and plotted as percentage rescue which was calculated as [(no. of colonies on glucose/no. of colonies on galactose) *100]. Plating assays were performed at least three times with comparable results which were quantitated.

\section{Halo Assay}

The background tester MATa strain (BJ3556) carrying the sst1-2 allele and the different GAL:CHC1 transformants were grown on YEP-glucose for 10-12 $\mathrm{h}$ and 16-20 $\mathrm{h}$ respectively. The tester strain was diluted to $0.1 \mathrm{OD}_{600}$ and spread onto YEP-glucose plate. Subsequently different GAL:CHC1 transformant cultures were spotted onto the plate which were incubated for 2-3 days before imaging. MATa and MAT $\alpha$ strains labelled in the Figure 4 are wild type strain controls.

\section{References}

1 Kaksonen, M. \& Roux, A. Mechanisms of clathrin-mediated endocytosis. Nature Reviews Molecular Cell Biology 19, 313 (2018).

2 Mayor, S. \& Pagano, R. E. Pathways of clathrin-independent endocytosis. Nature reviews Molecular cell biology 8, 603 (2007).

3 Bitsikas, V., Corrêa Jr, I. R. \& Nichols, B. J. Clathrin-independent pathways do not contribute significantly to endocytic flux. Elife 3, e03970 (2014).

4 Prosser, D. C., Drivas, T. G., Maldonado-Báez, L. \& Wendland, B. Existence of a novel clathrinindependent endocytic pathway in yeast that depends on Rho1 and formin. J Cell Biol 195, 657-671 (2011).

5 Seeger, M. \& Payne, G. S. A role for clathrin in the sorting of vacuolar proteins in the Golgi complex of yeast. The EMBO Journal 11, 2811-2818 (1992). 
Allen, C. L., Goulding, D. \& Field, M. C. Clathrin - mediated endocytosis is essential in Trypanosoma brucei. The EMBO journal 22, 4991-5002 (2003).

Lemmon, S. K. \& Jones, E. W. Clathrin requirement for normal growth of yeast. Science 238, 504-509 (1987).

Payne, G. S. \& Schekman, R. A test of clathrin function in protein secretion and cell growth. Science 230, 1009-1014 (1985).

Payne, G. S. \& Schekman, R. Clathrin: a role in the intracellular retention of a Golgi membrane protein. Science 245, 1358-1365 (1989).

10 Seeger, M. \& Payne, G. S. Selective and immediate effects of clathrin heavy chain mutations on Golgi membrane protein retention in Saccharomyces cerevisiae. The Journal of Cell Biology 118, 531-540 (1992).

1 Nelson, K. K., Holmer, M. \& Lemmon, S. K. SCD5, a suppressor of clathrin deficiency, encodes a novel protein with a late secretory function in yeast. Molecular biology of the cell 7, 245260 (1996).

Lemmon, S. K., Freund, C., Conley, K. \& Jones, E. W. Genetic instability of clathrin-deficient strains of Saccharomyces cerevisiae. Genetics 124, 27-38 (1990).

3 Moorthy, B. T., Sharma, A., Boettner, D. R., Wilson, T. E. \& Lemmon, S. K. Identification of Suppressor of Clathrin Deficiency-1 (SCD1) and Its Connection to Clathrin-Mediated Endocytosis in Saccharomyces cerevisiae. G3: Genes, Genomes, Genetics 9, 867-877 (2019).

14 Nelson, K. K. \& Lemmon, S. Suppressors of clathrin deficiency: overexpression of ubiquitin rescues lethal strains of clathrin-deficient Saccharomyces cerevisiae. Molecular and cellular biology 13, 521-532 (1993).

15 Rajyaguru, P., She, M. \& Parker, R. Scd6 targets elF4G to repress translation: RGG motif proteins as a class of elF4G-binding proteins. Molecular cell 45, 244-254 (2012).

16 Thandapani, P., O'Connor, T. R., Bailey, T. L. \& Richard, S. Defining the RGG/RG motif. Molecular cell 50, 613-623 (2013).

Ozdilek, B. A. et al. Intrinsically disordered RGG/RG domains mediate degenerate specificity in RNA binding. Nucleic acids research 45, 7984-7996 (2017).

Hilliker, A., Gao, Z., Jankowsky, E. \& Parker, R. The DEAD-box protein Ded1 modulates translation by the formation and resolution of an elF4F-mRNA complex. Molecular cell 43, 962-972 (2011).

Mitchell, S. F., Jain, S., She, M. \& Parker, R. Global analysis of yeast mRNPs. Nat Struct Mol Biol 20, 127-133, doi:10.1038/nsmb.2468 (2013).

Segal, S. P., Dunckley, T. \& Parker, R. Sbp1p affects translational repression and decapping in Saccharomyces cerevisiae. Molecular and cellular biology 26, 5120-5130 (2006).

Waldherr, M. et al. A multitude of suppressors of group II intron-splicing defects in yeast. Current genetics 24, 301-306 (1993).

22 Formosa, T. \& Nittis, T. Suppressors of the temperature sensitivity of DNA polymerase $\alpha$ mutations in Saccharomyces cerevisiae. Molecular and General Genetics MGG 257, 461-468 (1998).

3 lost, I., Dreyfus, M. \& Linder, P. Ded1p, a DEAD-box protein required for translation initiation in Saccharomyces cerevisiae, is an RNA helicase. Journal of Biological Chemistry 274, 1767717683 (1999).

4 Beckham, C. et al. The DEAD-box RNA helicase Ded1p affects and accumulates in Saccharomyces cerevisiae P-bodies. Molecular biology of the cell 19, 984-993 (2008).

25 Pang, T.-L., Wang, C.-Y., Hsu, C.-L., Chen, M.-Y. \& Lin, J.-J. Exposure of single-stranded telomeric DNA causes G2/M cell cycle arrest in Saccharomyces cerevisiae. Journal of Biological Chemistry 278, 9318-9321 (2003).

6 Windgassen, M. \& Krebber, $H$. Identification of Gbp2 as a novel poly (A)+ RNA - binding protein involved in the cytoplasmic delivery of messenger RNAs in yeast. EMBO reports 4 , 278-283 (2003).

7 Häcker, S. \& Krebber, H. Differential export requirements for shuttling serine/arginine-type mRNA-binding proteins. Journal of Biological Chemistry 279, 5049-5052 (2004). 
28 Hurt, E., Luo, M.-j., Röther, S., Reed, R. \& Sträßer, K. Cotranscriptional recruitment of the serine-arginine-rich (SR)-like proteins Gbp2 and Hrb1 to nascent mRNA via the TREX complex. Proceedings of the National Academy of Sciences 101, 1858-1862 (2004).

29 Hackmann, A. et al. Quality control of spliced mRNAs requires the shuttling SR proteins Gbp2 and Hrb1. Nature communications 5, 3123 (2014).

30 Windgassen, M. et al. Yeast shuttling SR proteins Npl3p, Gbp2p, and Hrb1p are part of the translating mRNPs, and Npl3p can function as a translational repressor. Molecular and cellular biology 24, 10479-10491 (2004).

31 Buchan, J. R., Muhlrad, D. \& Parker, R. P bodies promote stress granule assembly in Saccharomyces cerevisiae. The Journal of cell biology 183, 441-455 (2008).

32 Coller, J. \& Parker, R. General translational repression by activators of mRNA decapping. Cell 122, 875-886 (2005).

33 Mugler, C. F. et al. ATPase activity of the DEAD-box protein Dhh1 controls processing body formation. Elife 5, e18746 (2016).

34 Anantharaman, V. \& Aravind, L. Novel conserved domains in proteins with predicted roles in eukaryotic cell-cycle regulation, decapping and RNA stability. BMC genomics 5, 45 (2004).

35 Poornima, G., Shah, S., Vignesh, V., Parker, R. \& Rajyaguru, P. I. Arginine methylation promotes translation repression activity of elF4G-binding protein, Scd6. Nucleic acids research 44, 9358-9368 (2016).

36 Ghaemmaghami, S. et al. Global analysis of protein expression in yeast. Nature 425, 737 (2003).

37 Squirrell, J. M. et al. CAR-1, a protein that localizes with the mRNA decapping component DCAP-1, is required for cytokinesis and ER organization in Caenorhabditis elegans embryos. Molecular biology of the cell 17, 336-344 (2006).

38 Wilhelm, J. E., Buszczak, M. \& Sayles, S. Efficient protein trafficking requires trailer hitch, a component of a ribonucleoprotein complex localized to the ER in Drosophila. Developmental cell 9, 675-685 (2005).

39 Rao, B. S. \& Parker, R. Numerous interactions act redundantly to assemble a tunable size of P bodies in Saccharomyces cerevisiae. Proceedings of the National Academy of Sciences 114, E9569-E9578 (2017).

40 Krogan, N. J. et al. Global landscape of protein complexes in the yeast Saccharomyces cerevisiae. Nature 440, 637 (2006).

41 Huang, K. M. et al. Novel functions of clathrin light chains: clathrin heavy chain trimerization is defective in light chain-deficient yeast. Journal of cell science 110, 899-910 (1997).

42 Poornima, G. et al. RGG-motif self-association regulates elF4G-binding translation repressor protein Scd6. RNA biology (2019).

43 Marnef, A., Sommerville, J. \& Ladomery, M. R. RAP55: insights into an evolutionarily conserved protein family. The international journal of biochemistry \& cell biology 41, 977981 (2009).

44 Newpher, T. M., Idrissi, F.-Z., Geli, M. I. \& Lemmon, S. K. Novel function of clathrin light chain in promoting endocytic vesicle formation. Molecular biology of the cell 17, 4343-4352 (2006).

$45 \mathrm{Li}, \mathrm{X}$. et al. Different regulations of ROM2 and LRG1 expression by Ccr4, Pop2, and Dhh1 in the Saccharomyces cerevisiae cell wall integrity pathway. mSphere 1, e00250-00216 (2016). 
A

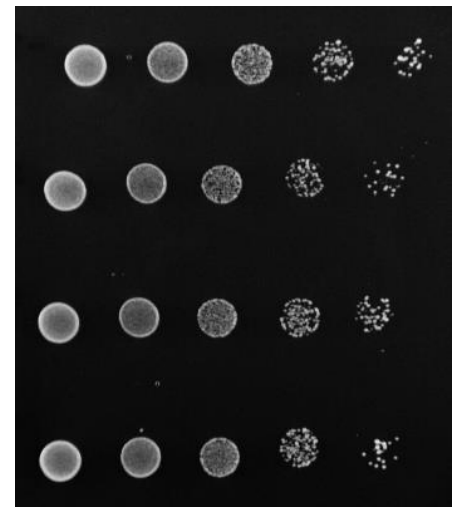

Galactose

B

C

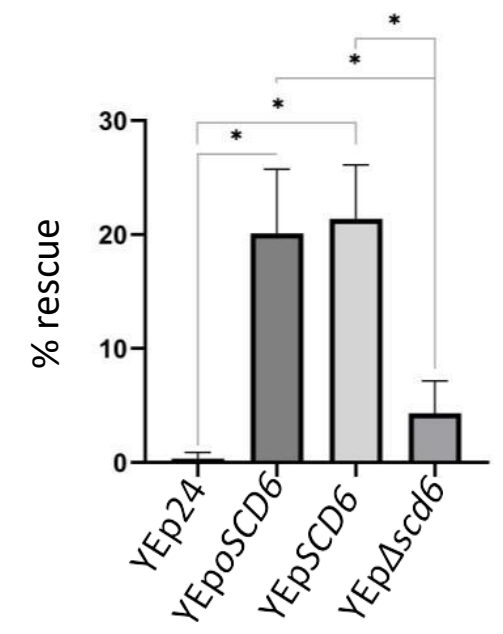

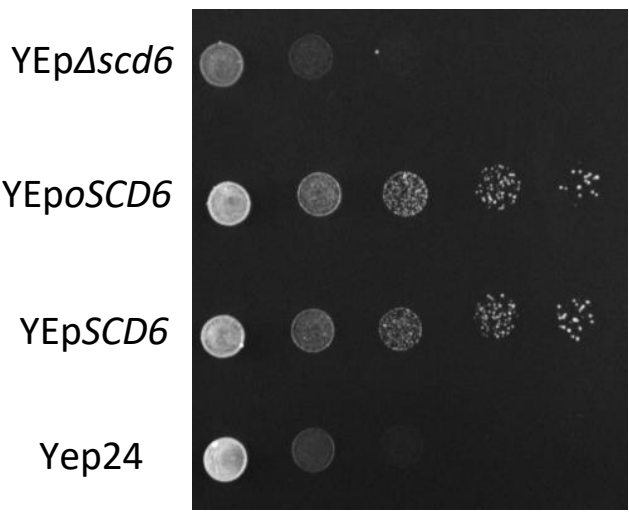

Glucose

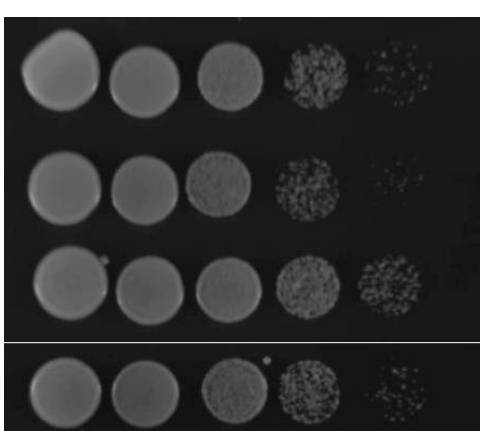

Galactose

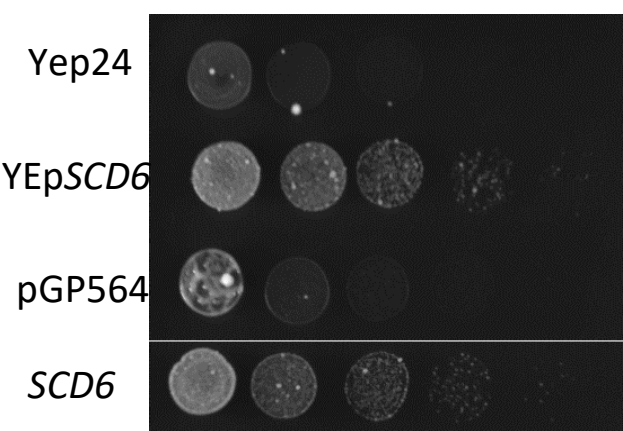

Glucose

Figure 1: YPR129W is necessary and sufficient to rescue clathrin deficiency

(A) Growth assay of GAL1:CHC1 cells (endogenous CHC1 is under galactose-inducible promoter) transformed with YEp24 (empty vector), YEpSCD6, YEpDscd6 (lacking YPR129W) and YEpoSCD6 (YEponlySCD6 expressing only YPR129W). $10 \mathrm{OD}_{600}$ cells were serially diluted and spotted on glucose or galactose containing selective media plates. These plates were incubated at $30^{\circ} \mathrm{C}$ for 2 days (galactose media) or 4-5 days (glucose media) before imaging. (B) Quantification of the percentage rescue of GAL1:CHC1 transformants upon clathrin deficiency (* indicates $p<0.05)$. Percentage rescue calculated as (number of colonies on glucose/number of colonies on galactose) *100. (C) Growth assay of GAL1:CHC1 transformants. SCD6 panel refers to the transformant expressing SCD6 from pGP564 vector with Cterminal His-tag. SCD6 expressed from pGP564 vector was spotted on the same plate as the rest of the three spottings. Image was spliced to remove other spottings not relevant to this figure. (Note: Because of the difference between the selection marker between Yep and pGP564, this assay was done on YEP plates). Plates were incubated at $30{ }^{\circ} \mathrm{C}$ for 2 days (galactose media) or $4-5$ days (glucose plate) before imaging. 


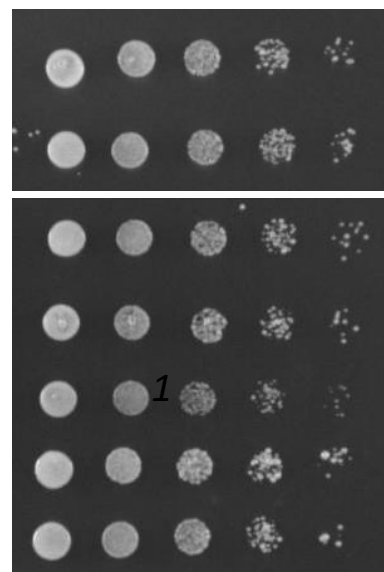

Galactose

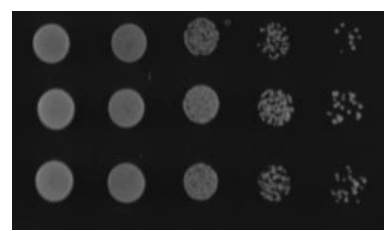

Galactose
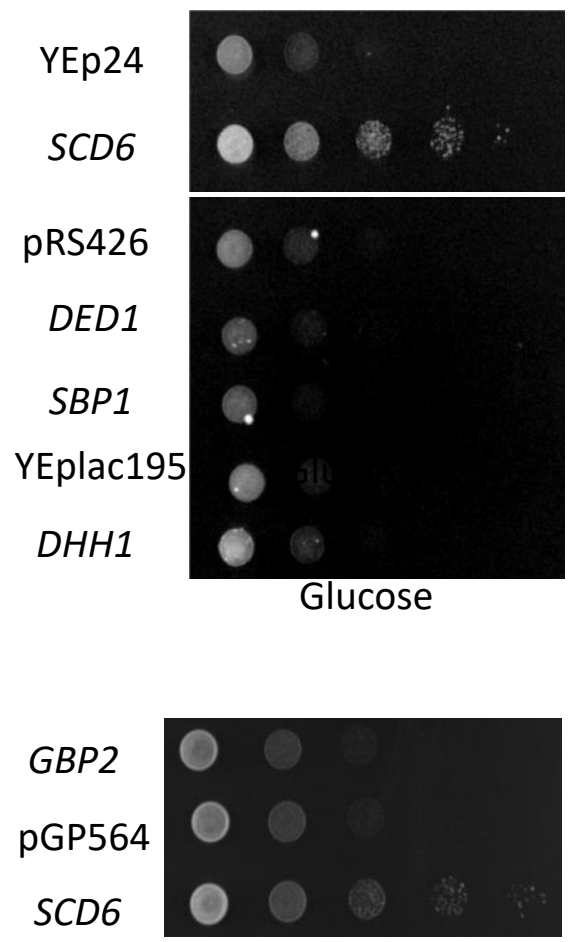

Glucose

B

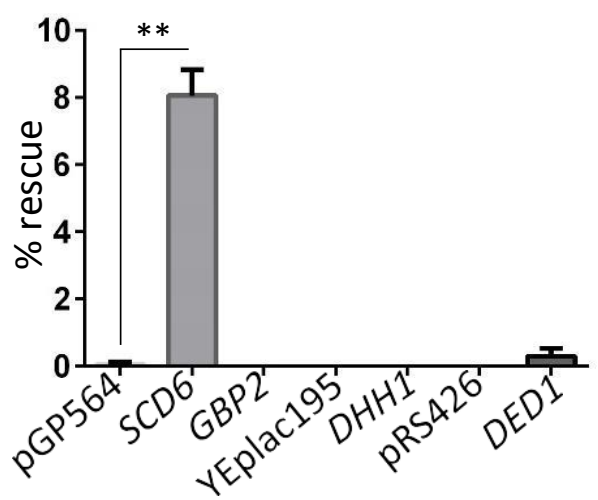

Figure 2: DED1, SBP1, DHH1 and GBP2 fail to rescue clathrin deficiency growth defect.

A) Growth assay of GAL1:CHC1 cells transformed with different plasmids as labeled. $10 \mathrm{OD}_{600}$ cells were serially diluted and spotted on glucose or galactose containing selective media plates. These plates were incubated at $30^{\circ} \mathrm{C}$ for 2 days (galactose media) or 4-5 days (glucose media) before imaging. YEp24 and SCD6 transformed cells were spotted on the same plate as the rest of the spottings. B) Quantification of plating assay to measure percentage rescue by GBP2, DED1 and $D H H 1$ ( ${ }^{* *}$ indicates $\mathrm{p}<0.01$ ). Percentage rescue was calculated as the number of colonies on glucose plate/number of colonies on galactose plate*100. 


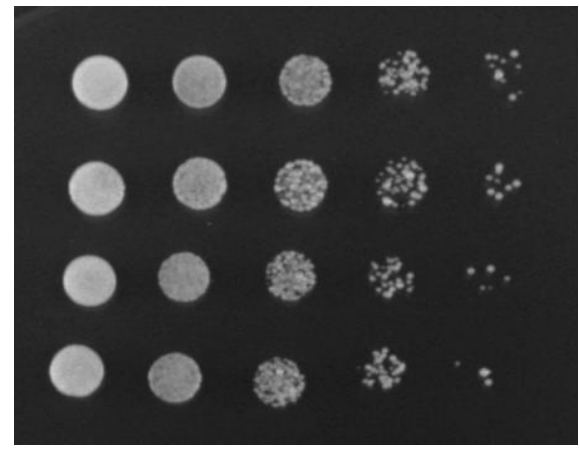

Galactose

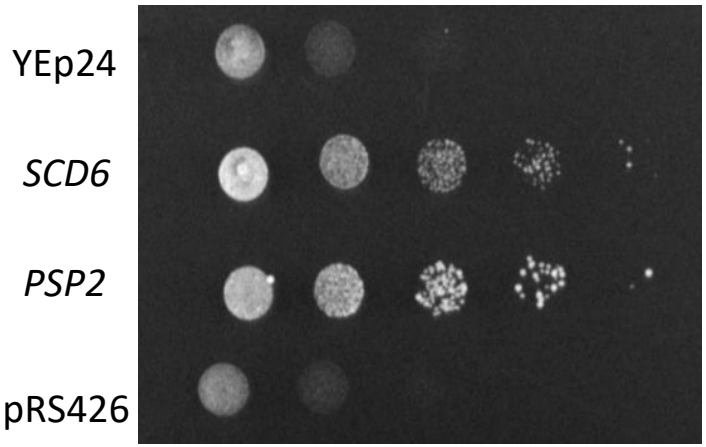

Glucose

B

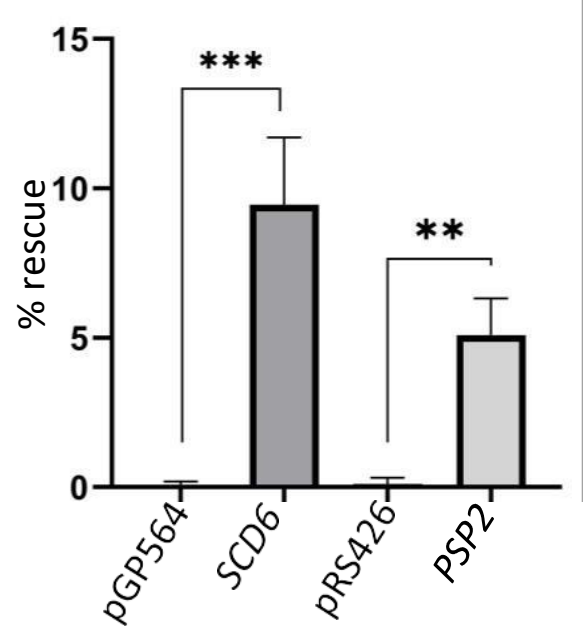

Figure 3: $P S P 2$ is a novel suppressor of clathrin deficiency

(A) GAL1:CHC1 cells were transformed with pRS426 (empty vector) or pRS426-PSP2. $10 \mathrm{OD}_{600}$ cells were serially diluted and spotted on glucose or galactose containing selective media plates. These plates were incubated at $30^{\circ} \mathrm{C}$ for 2 days (galactose media) or $4-5$ days (glucose media) before imaging. (B) Quantification of the percentage rescue by PSP2 upon clathrin deficiency using plating assay $(* *$ and $* * *$ indicates $p<0.01$ and $p<0.001$ respectively). Percentage rescue was calculated as the number of colonies on glucose plate/number of colonies on galactose plate*100. 


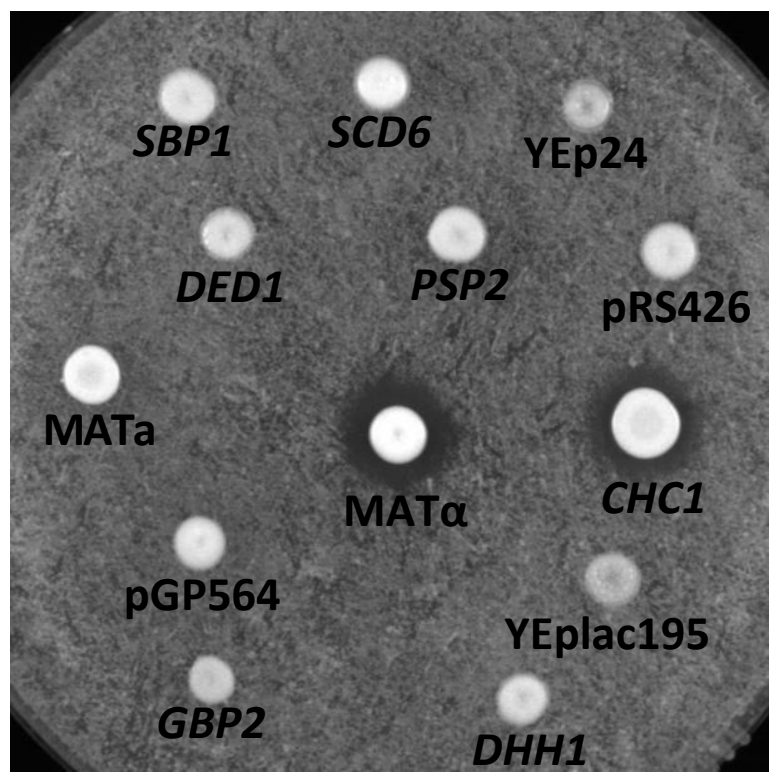

Figure 4: SCD6 and PSP2 fail to rescue the trans-Golgi network (TGN) sorting function of clathrin heavy chain.

MATa strain expressing endogenous $C H C 1$ under galactose-inducible promoter was transformed with plasmids expressing SCD6, SBP1, DED1, PSP2, GBP2, DHH1 or CHC1. Respective empty vectors were used as control. Transformants were spotted on a lawn of MATa cells carrying the sst1-2 allele and incubated on YEP glucose plate until (2-3 days) the appearance of a zone of clearance (halo). YEp24 is the empty vector control for SCD6 and CHC1. pRS426 is the empty vector control for DED1, SBP1 and PSP2. pGP564 is the empty vector control for GBP2 and YEplac195 is the empty vector control for DHH1. Wild type MATa and MAT $\alpha$ strains act as negative and positive controls, respectively. 
A

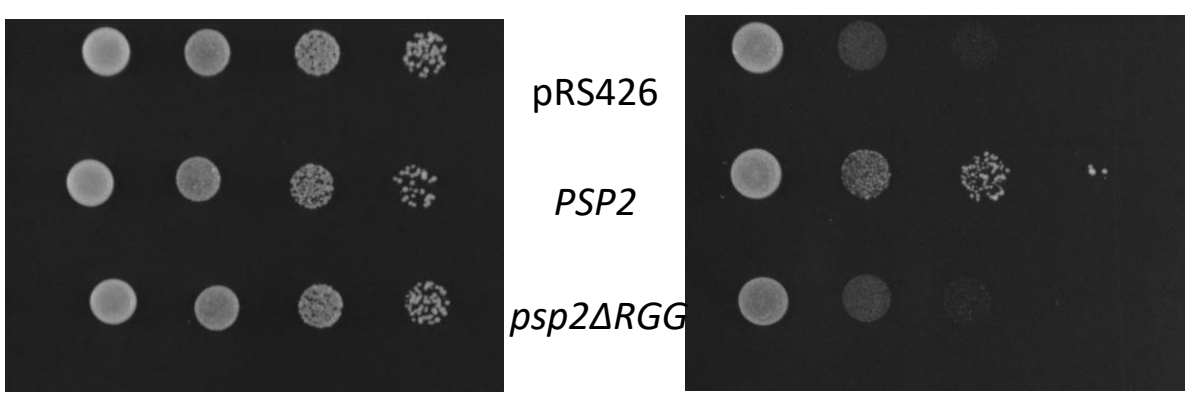

Galactose

Glucose

B

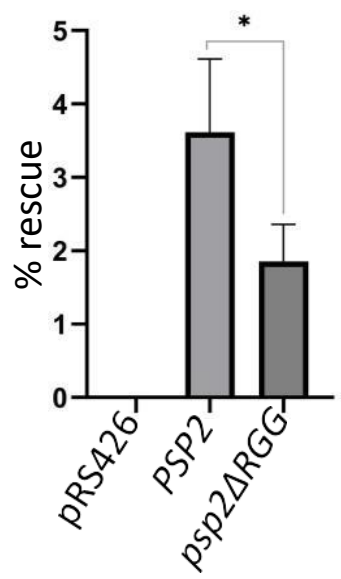

Figure 5: Deletion of RGG-motif compromises the ability of Psp2 to suppress clathrin deficiency growth defect.

(A) Growth assay of GAL1:CHC1 cells transformed with wild type and RGG-motif deletion mutants of Psp2. 10 $\mathrm{OD}_{600}$ cells were serially diluted and spotted on glucose or galactose containing selective media plates. These plates were incubated at $30^{\circ} \mathrm{C}$ for 2 days (galactose media) or $4-5$ days (glucose media) before imaging. (B) Quantification of the percentage rescue of GAL1:CHC1 transformants with wild type or RGG-motif deletion mutant of PSP2 upon clathrin deficiency (* indicates $p<0.05$ ). Percentage rescue was calculated as the number of colonies on glucose plate/number of colonies on galactose plate* 100. 
A

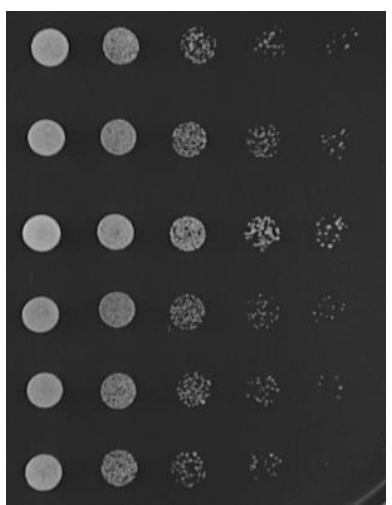

B

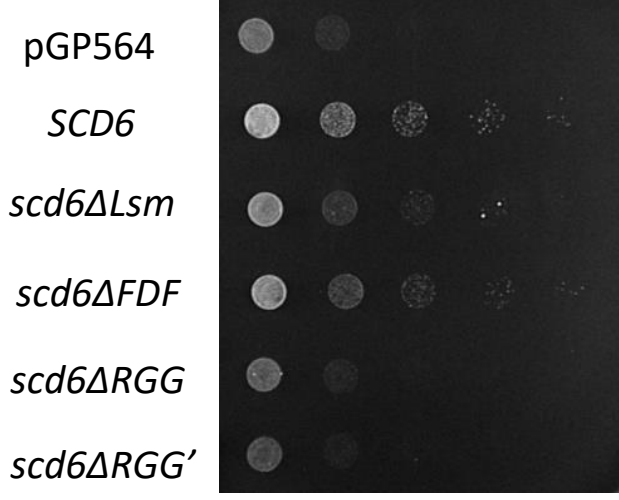

Glucose

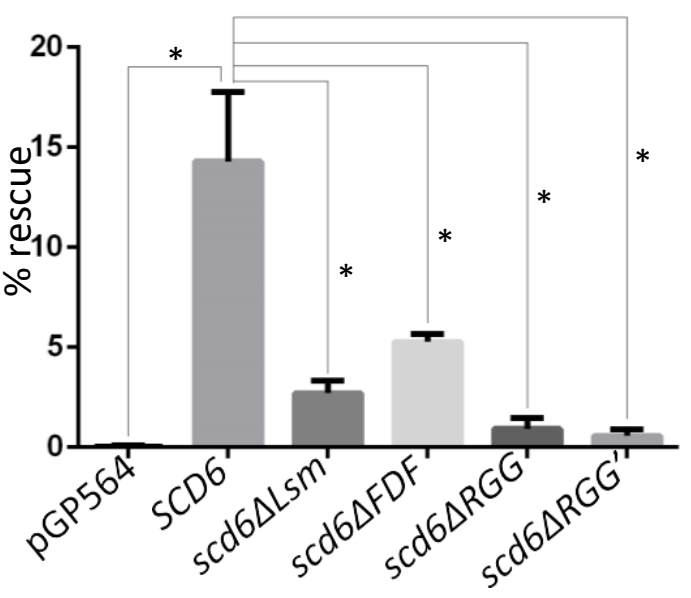

C

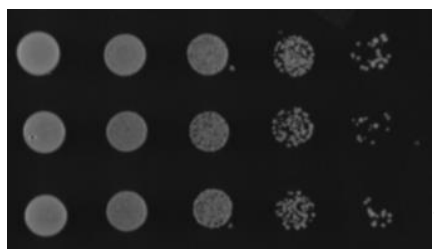

Galactose

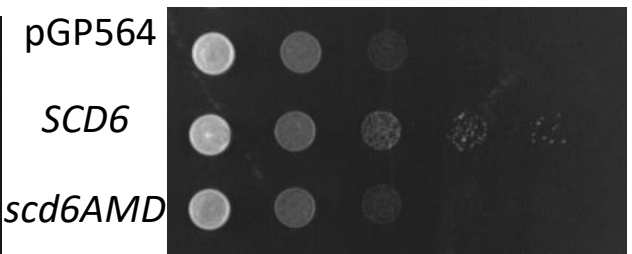

Glucose

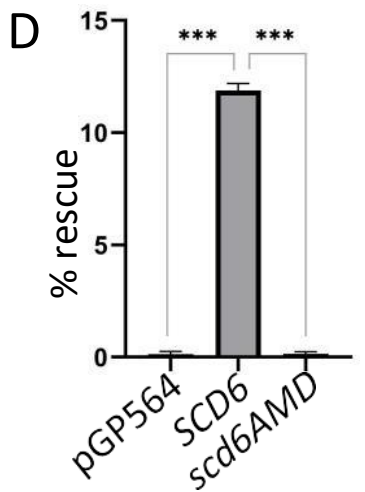

Figure 6: RGG-motif and Lsm domain are important for suppress clathrin deficiency growth defect by Scd6.

(A) Growth assay of GAL1:CHC1 cells transformed with plasmids expressing wild type, Lsm, FDF, RGG or RGG' domain/motif deletion mutants of Scd6. $10 \mathrm{OD}_{600}$ cells were serially diluted and spotted on glucose or galactose containing selective media plates. These plates were incubated at $30^{\circ} \mathrm{C}$ for 2 days (galactose media) or 4-5 days (glucose media) before imaging. (B) Quantification of the percentage rescue of GAL1:CHC1 by SCD6 mutants upon clathrin depletion. (C) Growth assay to test the ability of arginine methylation defective (AMD) mutant of SCD6 to suppressing clathrin deficiency. (D) Quantification of the percentage rescue of GAL1:CHC1 transformants by the AMD mutant of SCD6 upon clathrin deficiency (* and ${ }^{* *}$ indicates $p<0.05$ and $p<0.01$ respectively). Percentage rescue was calculated as the number of colonies on glucose plate/number of colonies on galactose plate*100. 
bioRxiv preprint doi: https://doi.org/10.1101/2020.03.11.986810; this version posted March 12, 2020. The copyright holder for this preprint (which was not certified by peer review) is the author/funder, who has granted bioRxiv a license to display the preprint in perpetuity. It is made available under aCC-BY-NC 4.0 International license. 